NBI-HE-93-36

August 1993

\title{
THE ROKHSAR-KIVELSON MODEL OF QUANTUM DIMERS AS A GAS OF FREE FERMIONIC STRINGS
}

\author{
Peter Orland ${ }^{*}$ 円 \\ The Niels Bohr Institute, \\ Blegdamsvej 17, DK-2100, \\ Copenhagen $\varnothing$, Denmark \\ orland@nbivax.nbi.dk \\ and \\ The City University of New York, \\ Baruch College, \\ 17 Lexington Ave., \\ New York, NY 10010, U.S.A. \\ orlbb@cunyvm.cuny.edu ${ }^{\dagger}$
}

\begin{abstract}
The $2+1$-dimensional quantum dimer model on a square lattice, proposed by Rokhsar and Kivelson as a theory of layered superconductivity, is shown to be equivalent to a many-body theory of free, transversely oscillating strings obeying Fermi statistics. A Jordan-Wigner construction for string field operators is presented. Topological defects are shown to be linearly confined in pairs by dynamical strings. Exact upper and lower bounds are placed on the ground-state energy and the string tension. It is argued that the system is in a spin-fluid phase and that there is no gap in the excitation spectrum.
\end{abstract}

\footnotetext{
$1^{*}$ Work supported by PSC-CUNY Research Award Program grant nos. 662467 and 663368 .

$2 \dagger$ Permanent address
} 


\section{Introduction}

In the last few years, progress has been made in exactly solving statistical and quantum models in a total of three dimensions. Some cases of the six-edge statistical model in three dimensions ${ }^{1}$, the $U(1)$ lattice gauge magnet ${ }^{2}$ and the quantum dimer model on a hexagonal lattice 3 in $2+1$ dimensions have been solved exactly.

In this article, the Rokhsar-Kivelson model (RKM) on the square lattice ${ }^{4}$ will studied from a similiar point of view. This system is a quantum dimer model on a square lattice, proposed as an effective theory of layered superconductors. It is found that, like the systems solved in references $1,2,3$, the RKM is a system of noninteracting Fermionic strings. While the one-string problem is not, unfortunately, exactly solvable, it can be understood physically without much effort. More general models with a "diagonal" coupling or with dynamical holons ${ }^{4}$ are not accessible to the techniques used here.

It is found here that the behaviour of the RKM without the "diagonal" term is qualitatively similiar to that of the quantum dimer model on the hexagonal lattice; topological defects (holons) are confined into pairs, but there is no gap in the spectrum and no long-range order. The ground state can be thus be thought of as a spin fluid. It can be shown that dimer configurations are the ground states of a particular Heisenberg model invented by Klein 5 . Presumably introducing further interactions in this Heisenberg model gives a system whose low-lying states are described by the RKM.

It is perhaps appropriate at this point to review some of the history and issues surrounding the RKM and related problems. Rokhsar and Kivelson's model has two parameters; a "resonance" coupling $J$ and a "diagonal" coupling $V$. These are coefficients of two different terms in the Hamiltonian. They were able to solve the model at $V=J$ where they found that the ground state was a superposition of every possible dimer state. For $V>J$ they showed that the ground state was a "spinstaggered" state with spontaneous breaking of rotational and translation invariance. They suggested that for $V<J$ a "spin-liquid" state with no long-range order should appear. They also argued that a "valence-bond-solid" or "column" phase should appear for $V<0$. In this phase so-called column configurations dominate, leading to a four-fold degeneracy of the ground state.Later, Dombré and Kotliar, who examined mean field theory for the Hubbard model and Read and Sachdev 6 , who studied $S U(N)$ generalizations of square-lattice Heisenberg antiferromagnets formally similiar to the RKM, did not find a spin-liquid phase, but did find a valence-bond-solid phase appeared ${ }^{7}$. The RKM model was then examined directly by Fradkin and Kivelson 8 who used dilute gas methods for the $V=0$ case and Sachdev, who studied the same problem numerically ${ }^{9}$. Fradkin and Kivelson argued that the $V=0$ ground

state was a valence-bond-solid state. Sachdev found that the susceptibility of the order parameter for valence-bond-solid formation was divergent with the volume, and therby suggested that such a solid formed for all $V<J$. Read and Sachdev later extended their analysis to antiferromagnets on hexagonal lattices and argued that 
valence-bond-solid formation took place there as well ${ }^{10}$. For at least some Heisenberg models, the ground state is a valence-bond solid ${ }^{11}$. In reference 3 , it was shown that a hexagonal-lattice quantum dimer model was in a gapless fluid phase ${ }^{12}$.

It will be shown here that the ground state of the square-lattice RKM at $V=0$ is a fluid and not a solid. The absence of long-range order is consistent with Rokhsar and Kivelson's original picture of superconductivity. There do appear to be power-law correlations which may explain Sachdev's results 9 .

In this paper, the string formulation is arrived at through the use of a generalization of the Jordan-Wigner transformation due to Dotsenko and Polyakov for the three-dimensional Ising model 13 . In previous solutions $1,2,3$ antisymmetrized string wave functions were used to diagonalize the Hamiltonian. The main reason for presenting the more formal Jordan-Wigner construction here is that some readers may find it more straightforward as well as more interesting from a mathematical point of view.

The RKM will first be converted to a triangular-lattice problem which resembles the neutral $U(1)$ lattice gauge magnet $2,14,15$. Next, the Hilbert space will be mapped into that of strings with an infinitely short-range, infinitely repulsive interaction. The interaction is removed by quantizing the strings as Fermions. The eigenfunctions and eigenvalues of the RKM can thereby be written in terms of the one-string eigenvectors and eigenvalues. The one-string problem is not exactly solvable, but physical arguments indicate its low-lying spectrum is that of a $1+1$-dimensional massless quantum field theory of Dirac particles. It is argued that the spectrum of the full RKM is gapless. A linear potential exists between topological defects, due to the formation of dynamical strings. Exact upper and lower bounds are found on both the ground-state energy and the string tension.

\section{The Rokhsar-Kivelson Model}

The sites of a two-dimensional lattice will be labeled by pairs of integers $\left(y^{1}, y^{2}\right)=\mathbf{y}$ and bonds $(\mathbf{y}, i)$ connecting $\mathbf{y}$ to $\mathbf{y}+\hat{\mathbf{i}}$. A spin state $|s(\mathbf{y}, i)\rangle$ is defined on each bond, by $s(\mathbf{y}, i)=1 / 2$ when a dimer is present at $(\mathbf{y}, i)$ and $s(\mathbf{y}, i)=-1 / 2$, when no dimer is present at $(\mathbf{y}, i)$. A dimer represents a $\pi$-valence bond between two nearest-neighbor copper atoms 16 . The Hamiltonian of the RKM is 4

$$
H=J \sum_{\mathbf{y}} \sum_{ \pm} \sigma^{ \pm}(\mathbf{y}, 1) \sigma^{\mp}(\mathbf{y}+\hat{\mathbf{1}}, 2) \sigma^{ \pm}(\mathbf{y}+\hat{\mathbf{2}}, 1) \sigma^{\mp}(\mathbf{y}, 2) .
$$

Here $\sigma^{ \pm}(\mathbf{y}, i)=(1 / 2)\left(\sigma^{x}(\mathbf{y}, i) \pm i \sigma^{y}(\mathbf{y}, i)\right)$, where $\sigma^{x}, \sigma^{y}$ and $\sigma^{z}$ are the usual Pauli matrices. The Hamiltonian density applied to a plaquette (square) changes that plaquettes's state according to the rule:

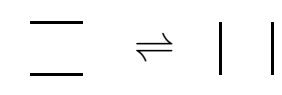


with all other states annihilated. The RKM is a $U(1)$ lattice gauge theory $2,14,15 ; H$ commutes with the Gauss' law operator defined at a site $\mathbf{y}$, adjacent to four links $l$

$$
G(\mathbf{y})=\sum_{l} \sigma^{z}(l)
$$

Dimer states $\mid \Psi>$ satisfy

$$
[G(\mathbf{y})+2] \mid \Psi>=0 .
$$

The Hamiltonian is identical to that of the model discussed in reference 2, though the Gauss' law condition (2) is different. The gauge-invariant Wilson loop operator defined on a closed contour of consecutive links $C=\left\{l_{1}, l_{2}, \ldots, l_{P}\right\}$ is

$$
A(C)=\sigma^{+}\left(l_{1}\right) \sigma^{-}\left(l_{2}\right) \sigma^{+}\left(l_{3}\right) \ldots \sigma^{-}\left(l_{P}\right) .
$$

A topological defect is a site $\vec{y}$ at which no dimers are attached. Such defects are characterized by

$$
[G(\mathbf{y})+4] \mid \Psi>=0 .
$$

In reference 4 a second "diagonal" term was added to the Hamiltonian, namely

$$
H_{d}=-V \sum_{\mathbf{y}} \mathcal{S}^{ \pm}(\mathbf{y}, 1) \mathcal{S}^{\mp}(\mathbf{y}+\hat{\mathbf{1}}, 2) \mathcal{S}^{ \pm}(\mathbf{y}+\hat{\mathbf{2}}, 1) \mathcal{S}^{\mp}(\mathbf{y}, 2)
$$

where $\mathcal{S}^{ \pm}$is defined by

$$
\mathcal{S}^{ \pm}(\mathbf{y}, i)=\frac{1 \pm \sigma^{z}(\mathbf{y}, i)}{2}
$$

In this paper, only the $V=0$ case will be considered.

\section{Reduction to a Triangular Lattice}

The RKM can be reduced to a spin model on a triangular lattice. The triangular lattice will be drawn as a square lattice with extra diagonal bonds. Upon making this reduction, $90^{\circ}$ rotational invariance is no longer manifest. Consider the bonds $(\mathbf{y}, 2)$, with $y^{1}+y^{2}$ odd. These are half the bonds parallel to $\hat{2}$. The occupation state at each of these bonds is a redundant degree of freedom. All such bonds may be contracted to points, leaving a triangular lattice. To see this, consider the possible dimer configurations around one such bond:

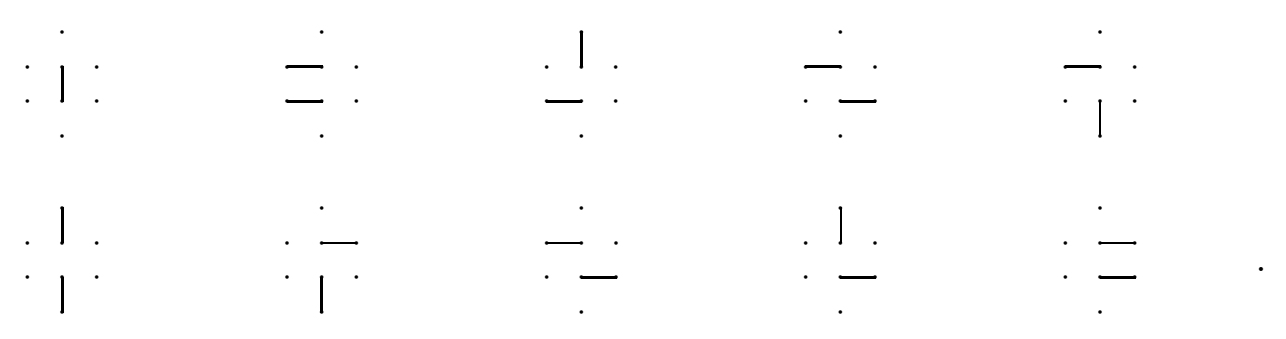


The middle bond is now contracted to a point (Fig.1). The sites of the triangular lattice will be labeled by $\mathbf{x}=\left(x_{1}, x_{2}\right)$ and the bonds connecting $\mathbf{x}$ to $\mathbf{x}+\hat{\mathbf{1}}$, connecting $\mathbf{x}$ to $\mathbf{x}+\hat{\mathbf{2}}$ and connecting $\mathbf{x}$ to $\mathbf{x}-\hat{\mathbf{1}}-\hat{\mathbf{2}}$ by $(\mathbf{x}, 1),(\mathbf{x}, 2)$ and $(\mathbf{x}, 3)$ respectively. The state at each bond is represented by a spin $\mid s(\mathbf{x}, i)>$. Next apply the unitary transformation which flips the spins at all horizontal bonds $\prod_{\mathbf{x}} \sigma^{x}(\mathbf{x}, \mathbf{1})$ (i.e. occupied bonds at $(\mathbf{x}, 1)$ are now replaced by empty bonds and vice-versa). The possible configurations at a site of the triangular lattice are now:
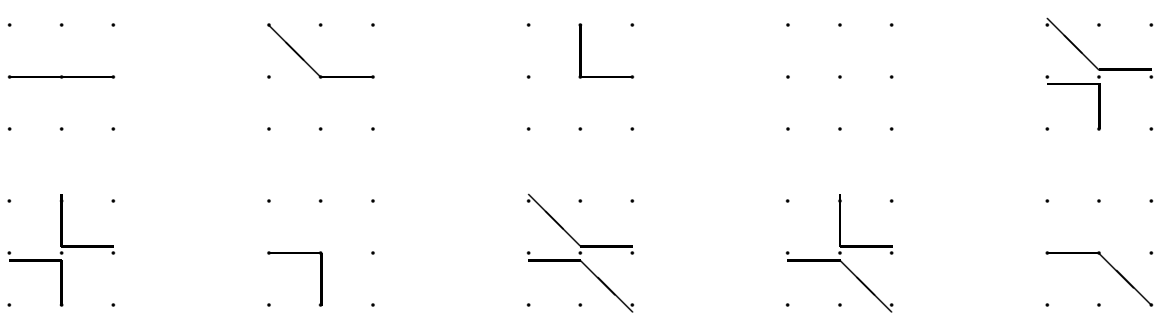

respectively. The fifth, sixth, eighth and ninth configurations have purposely been drawn with a break. The new Hilbert space is restricted by the Gauss law

$$
g(\mathbf{x}) \mid \Psi>=0
$$

where

$$
g(\mathbf{x})=\sigma^{z}(\mathbf{x}, 1)-\sigma^{z}(\mathbf{x}, 2)+\sigma^{z}(\mathbf{x}, 3)-\sigma^{z}(\mathbf{x}-\hat{\mathbf{1}}, 1)+\sigma^{z}(\mathbf{x}-\hat{\mathbf{2}}, 2)-\sigma^{z}(\mathbf{x}+\hat{\mathbf{1}}+\hat{\mathbf{2}}, 3) .
$$

The Hamiltonian is now

$$
\begin{aligned}
H & =\sum_{\mathbf{x}} \sum_{ \pm}\left[\sigma^{ \pm}(\mathbf{x}, 1) \sigma^{ \pm}(\mathbf{x}, 2) \sigma^{\mp}(\mathbf{x}+\hat{\mathbf{2}}, 3)\right. \\
& \left.+\sigma^{ \pm}(\mathbf{x}+\hat{\mathbf{2}}, 3) \sigma^{\mp}(\mathbf{x}+\hat{\mathbf{2}}, 1) \sigma^{\mp}(\mathbf{x}+\hat{\mathbf{1}}, 2)\right],
\end{aligned}
$$

This $H$ commutes with $g(\mathbf{x})$ defined in (9). It interchanges states on triangles according to:

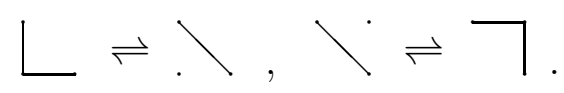

A typical configuration of the square lattice is shown in Fig. 2. The basis states of the Hilbert space are strings extending across the lattice. Two strings never overlap on any link.

The strings are not arbitrary paths. Any unit segment of string on a vertical bond (that is, a bond in the 2-direction) or on a bond at a $45^{\circ}$ (that is, a bond of string parallel to $\hat{\mathbf{1}} \mathbf{-} \hat{\mathbf{2}}$ ) must be attached at the top to a segment of string on a 
horizontal bond (in the 1-direction), coming from the left, unless the vertical bond is on the boundary. In addition, a segment of string on a vertical or $45^{\circ}$ bond must be attached at the bottom to a segment of string on a horizontal bond, going off to the right. Three impossible configurations for two adjacent segments of a single string are:
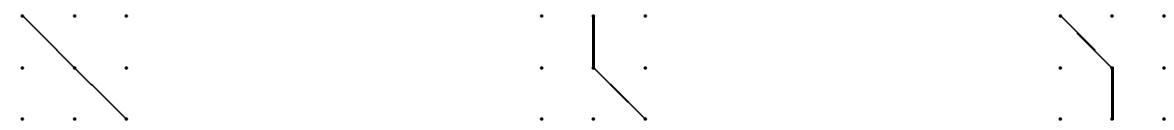

The strings interact though a short-range, infinitely strong repulsive interaction at links. Systems of Bosonic strings with such "hard-core" repulsion in $2+1$ dimensions are equivalent to systems of free Fermionic strings 2,3 .

Assume the original lattice of the RKM has the shape of a rectangle of vertical dimension $2 N$ and horizontal dimension $L$, where $L$ is odd. Select for contraction those links $(\mathbf{y}, 2)$ for which $y^{1}+y^{2}$ is an odd number. Then the resulting triangular lattice has the appearance shown at the bottom of Fig.1. It is possible to see that all but one site of the left boundary $x^{1}=1$ of the triangular lattice is an endpoint of exactly one string. There are therefore $N$ strings attached to this boundary (it contains $N+1$ sites). The site which is not an endpoint of a string is that at the bottom of the left boundary (it is possible for a string to pass though this site, but not end there). Similiarly, all but one site of the right boundary $x^{1}=L$ of the triangular lattice is an endpoint of exactly one string, the exception being the site at the top of the right boundary. The number of strings is conserved by the Hamiltonian. With this choice of boundary condition, the top and bottom boundaries place no restriction on the shape a string may take.

If the system were in a valence-bond-solid phase, the ground state would be spontaneously broken. It would be dominated by one of the four column configurations shown in Fig.3. On the triangular lattice the column configurations become those shown in Fig.4. It will be argued here, that such symmetry breaking does not take place (provided there is no diagonal term (6) included in the Hamiltonian). The argument hinges on the string spectrum being gapless. In fact, a much simpler argument can be made against valence-bond-solid formation. Consider any plaquette containing two dimers in a column configuration. The state of this plaquette is completely free to fluctuate. There is no preference for the two dimers on this plaquette to be aligned along the 1-direction or the 2-direction. The configuration of each of the other plaquettes containing two dimers can also fluctuate. The solid cannot maintain its integrity and must melt.

\section{The One-String Problem}

Each string is made of segments attached end-to-end. A string configuration is described by a sequence of numbers $X(k)$, each an integer or half-integer, defined below. 
The midpoint of each segment has 1 -coordinate $x^{1}=n / 2+1 / 2, n$ even, while the endpoints have 1 -coordinates $x^{1}=n / 2+1 / 2, n$ odd. In this way, both midpoints and endpoints of segments are labeled by the horizontal coordinate $n$. The number $X(k)$ is an integer or half-integer equal to the value of $x^{2}$ at the midpoint of the segment of string at $x^{1}=k+1 / 2$. Clearly $n=2 k$. The eigenstates of the one-string Hamiltonian in the Schrödinger picture are wave functionals $S[X]=S[X(1), \ldots, X(k), \ldots, X(L)]$. Though the links $(\mathbf{x}, \mathbf{1})$ with $x^{1}=L$ do not exist on the triangular lattice, the number $X(L)$ is still needed. The purpose of $X(L)$ is to specify how the string ends at the right boundary. These string wave functionals vanish for any choice of $[X]$ which does not correspond to a string. Define the raising and lowering operators on this space of wave-functionals by

$$
\left[\Delta(k), X\left(k^{\prime}\right)\right]=\delta_{k, k^{\prime}}, \quad\left[\Delta^{\dagger}(k), X\left(k^{\prime}\right)\right]=-\delta_{k, k^{\prime}}
$$

The string configuration is next mapped into a one-dimensional spin configuration $\{s(n)\}, s(n)= \pm 1$. A spin at odd $n$ is down unless the string segment at $x^{1}=n / 2$ is parallel to $\hat{\mathbf{2}}$. A spins at even $n$ is down, unless the string segment whose midpoint is $x^{1}=n / 2$ is tilted at $45^{\circ}$, i.e. parallel to $\hat{\mathbf{1}}-\hat{\mathbf{2}}$. The one-string Hamiltonian is equivalent to a spin-chain Hamiltonian under an inner-product-preserving transformation A:

$$
\mathcal{H}=A h A^{\dagger}
$$

defined by

$$
\begin{aligned}
A \sigma^{z}(2 l-1) A^{\dagger} & =2 \delta_{X(l), X(l-1)-1}-1, \\
A \sigma^{z}(2 l) A^{\dagger} & =2 \delta_{X(l+1), X(l)-1 / 2} \delta_{X(l), X(l-1)-1 / 2}-1, \\
A \sigma^{+}(2 l-1) A^{\dagger} & =\prod_{m=2 l}^{2 L+1} \Delta(m)^{2} \\
A \sigma^{+}(2 l) A^{\dagger} & =\Delta(2 l) \prod_{m=2 l+1}^{2 L+1} \Delta(m)^{2}, \\
A \sigma^{-}(2 l-1) A^{\dagger} & =\prod_{m=2 l}^{2 L+1} \Delta^{\dagger}(m)^{2} \\
A \sigma^{-}(2 l) A^{\dagger} & =\Delta^{\dagger}(2 l) \prod_{m=2 l+1}^{2 L+1} \Delta^{\dagger}(m)^{2} .
\end{aligned}
$$

Certain restrictions must be placed on spin configurations if they are to be identified by (13) with string configurations. They are:

1. the spins $s(n)$ and $s(n+1)$ cannot both be +1 ,

2. the spins $s(2 l)$ and $s(2 l+2)$ cannot both be +1 , 
The spin-chain Hamiltonian which describes the string is

$$
h=J \sum_{n=1}^{2 L} \sigma_{n}^{ \pm} \sigma_{n+1}^{\mp}+\lambda \sum_{n=1}^{2 L}\left(1+\sigma_{n}^{z}\right)\left(1+\sigma_{n+1}^{z}\right)+\lambda \sum_{l=1}^{L-1}\left(1+\sigma_{2 l}^{z}\right)\left(1+\sigma_{2 l+2}^{z}\right),
$$

where $\lambda$ is taken to infinity to enforce the above restrictions 1 and 2. Notice that the last term breaks the invariance under translation by one lattice spacing (this invariance is also broken by the boundary conditions). The number of up spins is fixed by virtue of the fact that the spin chain has a global $U(1)$ invariance:

$$
\left[h, \sum_{n=1}^{2 L+1} \sigma_{n}^{z}\right]=0
$$

The Hamiltonian (14) describes spinless Fermions which can hop from site to site, with strong nearest-neighbor and next-to-nearest-neighbor repulsion. The Fermion fields are constructed through the Jordan-Wigner transformation 17 :

$$
\psi_{n}^{\dagger}=\sigma_{n}^{+} \prod_{m<n} \sigma_{m}^{z}, \quad \psi_{n}=\sigma_{n}^{-} \prod_{m<n} \sigma_{m}^{z}
$$

These obey $\left[\psi_{n}^{\dagger}, \psi_{m}\right]_{+}=\delta_{n, m}$, with all other anticommutators vanishing. The filling fraction must be $1 / 4$ by virtue of the boundary conditions (this is consistent with (15) ). Fermions seperated from each other by more than two lattice spacings have the usual hopping dispersion relation $E_{k}=2 J \cos k$, hence a Fermi sea forms in the ground state of (14). If the second term in (14) is ignored, the low-lying excitations are one-dimensional massless Dirac particles ${ }^{17}$. It will be argued that this is true for the system (14) as well.

The additional repulsive interactions in (14) should not produce a gap above the Fermi sea. There are two reasons why this should be so. The first of these is that a repulsive short-range interaction cannot form bound states in the Fermi fluid. Secondly, the spin-chain is parity-invariant (in the sense that a spin at a given site is coupled in the same fashion to its left neighbor as its right neighbor), so that the left and right Dirac sectors should be uncoupled, which is inconsistent with a gap. However, I do not have a proof of this claim.

The Hamiltonian (14) cannot be solved exactly, but it should be possible to study its spectrum by numerical methods. It is possible, however, to find exact upper and lower bounds for its ground-state energy. While this can actually be done for the finite open chain, I will do so only for the ground-state energy per unit length in the thermodynamic limit.

For simplicity, assume that the Hamiltonian (14 is put on lattice of length $\mathcal{L}$ with periodic boundary conditions, i.e. $\sigma_{n+\mathcal{L}}^{i}=\sigma_{n}^{i}$. Consider the Hamiltonian

$$
h_{R}=J \sum_{n=1}^{\mathcal{L}} \sigma_{n}^{ \pm} \sigma_{n+1}^{\mp}+\lambda \sum_{n=1}^{\mathcal{L}} \sum_{j=1}^{R}\left(1+\sigma_{n}^{z}\right)\left(1+\sigma_{n+j}^{z}\right)
$$


on such a lattice and where, as before, $\lambda$ is taken to infinity. The number $R$ is the range of the potential and can be any non-negative integer (The $R=0$ case is the XX chain $\left.{ }^{17}\right)$. For a specified filling fraction $f=\mathcal{N} / \mathcal{L}$, the ground-state energy per site of $h_{1}$ and $h_{2}$ will be shown to be lower and upper bounds, respectively, of the groundstate energy per site of $h$. In addition the ground-state energy per site of $h_{R}$ can be explicitly calculated for any filling fraction. This model was considered recently by Gómez-Santos, who noted that for particular fillings its solution is a Luttinger liquid 18 .

It is straightforward to find all the eigenstates and eigenvalues of $h_{R}$. Consider the one-up-spin eigenstates. These states are labeled by wave number $k=2 \pi p / \mathcal{L}$ where $p=0, \ldots, \mathcal{L}-1$ :

$$
\left|k>=\sum_{n=1}^{\mathcal{L}} e^{i k n} \sigma_{n}^{+}\right| \Omega>,
$$

where $\mid \Omega>$ is the state with all spins down (zero filling fraction). These states are eigenstates of both $h$ and $h_{R}$ with eigenvalues $E_{k}=2 \cos k$. The eigenstates of $h$ and $h_{R}$ must have the form

$$
\begin{aligned}
\mid k_{1}, \ldots, k_{\mathcal{N}}> & =\left(\sum_{n}\right)^{\prime} \exp \left(i k_{1} n_{1}+\cdots+i k_{\mathcal{N}} n_{\mathcal{N}}\right) \\
& \times \Psi_{k}\left(n_{1}, \ldots, n_{\mathcal{N}}\right) \sigma_{n_{1}}^{+} \cdots \sigma_{n_{\mathcal{N}}}^{+} \mid \Omega>
\end{aligned}
$$

where the sum is over $1 \leq n_{1}<n_{2}<\cdots<n_{\mathcal{N}} \leq \mathcal{L}$. A state of the form (19) is an eigenstate of $h_{R}$ if and only if it is an eigenstate of the XX model (free Fermions) and satisfies the condition that $\Psi_{k}\left(n_{1}, \ldots, n_{\mathcal{N}}\right)=0$ if for some $r, n_{r+1}-n_{r} \leq R$. Thus any eigenstate of $h_{R+1}$ is also an eigenstate of $h_{R}$ with the same eigenvalue. A state of the form (19) is an eigenstate of $h$ if and only if it is an eigenstate of the $X X$ model and satisfies the condition that $\Psi_{k}\left(n_{1}, \ldots, n_{\mathcal{N}}\right)=0$ if either :

1. $n_{r+1}-n_{r} \leq 1$, for any $r$,

2. $n_{2 p+1}-n_{2 p} \leq 2$, for any $p$.

These conditions imply that any eigenstate of $h_{2}$ is also an eigenstate of $h$ and any eigenstate of $h$ is also an eigenstate of $h_{1}$. Therefore the ground state at filling fraction $f$ of $h_{2}$ is an eigenstate of $h$ and the ground state at filling fraction $f$ of $h$ is an eigenstate of $h_{1}$. If $\epsilon_{R}(f)$ is the ground-state energy per site of $h_{R}$ and $\epsilon(f)$ is the ground-state energy per site of $h$, then the above considerations imply

$$
\epsilon_{1}(f) \leq \epsilon(f) \leq \epsilon_{2}(f)
$$

The simplest way to calculate $\epsilon_{R}(f)$ is to notice that $h_{R}$ is just a free Fermion problem (XX chain) on an effective lattice of size $\mathcal{L}^{\prime}=\mathcal{L}-\mathcal{N} R=\mathcal{L}(1-f R)$. The effective filling fraction is

$$
f^{\prime}=\frac{\mathcal{N}}{\mathcal{L}^{\prime}}=\frac{f}{1-f R}
$$


Now $\mathcal{L}^{\prime} \epsilon_{0}\left(f^{\prime}\right)$ is by definition, the ground-state energy of the free Fermion problem on a lattice of size $\mathcal{L}^{\prime}$ and filling fraction $f^{\prime}$. The ground-state energy per site of $h_{R}$ at filling fraction $f$ must therefore be

$$
\epsilon_{R}(f)=\frac{\mathcal{L}^{\prime} \epsilon_{0}\left(f^{\prime}\right)}{\mathcal{L}}=(1-f R) \epsilon_{0}\left(\frac{f}{1-f R}\right) .
$$

The ground-state energy density the free Fermi problem on a large lattice with this filling fraction is

$$
\epsilon_{0}\left(\frac{f}{1-f R}\right)=-\frac{2 J}{\pi} \sin \left(\frac{\pi f}{1-f R}\right) .
$$

In the thermodynamic limit, the energy per site of the string on a lattice on dimensions $N \times L$ is given by $\mu=\epsilon(1 / 4) / 2$. From (22) and (23) the inequality (20) becomes

$$
-\frac{1}{\sqrt{3} \pi} J \leq \mu \leq-\frac{1}{\pi} J
$$

or

$$
-0.184 J \leq \mu \leq-0.159 J
$$

\section{The $N$-String Problem}

The solution of the $N$-string problem is essentially the same as in references 2 and 3. First the one-string Hamiltonian is found. Then the product of $N$ one-string eigenfuntionals is antisymmetrized in each $X(n)$. This gives an eigenfunctional of $\mathcal{H}_{1}+\ldots+\mathcal{H}_{N}$, where $\mathcal{H}_{k}$ is the Hamiltonian for the $k^{\text {th }}$ string. It is in fact an eigenfunctional of the full Hamiltonian, since it is guaranteed to vanish whenever strings overlap.

Antisymmetrization can "break up" some of the strings; it leads to choices of $[X]$ which are not continuously connected strings. The wave functionals automatically vanish whenever this is the case.

Let $\mathbf{S}_{N}$ be the permutation group of $N$ objects. Given $N$ strings, there are $N$ values of $X(k)$ for each $k$, which will be labeled $X^{1}(k), \ldots, X^{N}(k)$. Permutations $s_{k} \varepsilon \mathbf{S}_{N}$ will act by sending the values of $X(k)$ to $X^{s_{1}(1)}(k), \ldots, X^{s_{k}(N)}(k)$. The antisymmetrized product of $N$ string wave functionals quantum numbers (which are the momenta of Fermions in each string) $\{\theta\}_{j}$ for $j=1, \ldots, N$ is

$$
S_{\{\theta\}_{1} \ldots\{\theta\}_{N}}[X]=\left[\prod_{k=1}^{L} \frac{1}{N !} \sum_{s_{k} \varepsilon \mathbf{S}_{N}} \operatorname{sgn}\left(s_{k}\right)\right] \prod_{j=1}^{N} S_{\{\theta\}_{j}}\left[X^{s_{1}(j)}(1), \ldots, X^{s_{L}(j)}(L)\right] .
$$

The argument $[X]$ is then restricted to a fundamental region $\mathcal{F}$ in which there is no

overlapping of strings:

$$
X^{1}(k)<X^{2}(k)<\ldots<X^{N}(k)
$$


This proceedure is consistent because $S_{\{\theta\}_{1} \ldots\{\theta\}_{N}}[X]$ vanishes just outside $\mathcal{F}$, where links from different strings touch. With this restriction, $\mathcal{H}_{1}+\ldots+\mathcal{H}_{N}$ can be identified with $\mathcal{H}_{s}$, and hence with the RKM Hamiltonian and $S_{\{\theta\}_{1} \ldots\{\theta\}_{N}}[X]$ are indeed the correct eigenstates. The energy eigenvalues are

$$
\mathcal{E}_{\{\theta\}_{1}, \ldots,\{\theta\}_{N}}=E_{\{\theta\}_{1}}+\ldots+E_{\{\theta\}_{N}} .
$$

The reader who is not entirely convinced of the results of this section can find a more complete discussion of many-string theory in reference 3 .

\section{String Field Theory}

A more formal method of converting the RKM to a many-string problem is the stringJordan-Wigner transformation, similiar to that discussed for the three-dimensional Ising model 13 .

Let $[X]=[X(1), \ldots, X(k), \ldots, X(L)]$ be a set of links $l_{1}, l_{2}, \ldots$ connecting the left boundary to the right boundary. These links do not neccesarily constitute a string configuration consistent with the rules discussed in Section 3, but are assumed to be a connected path, such that, for each $k=1, \ldots, L$ there is a unique $X(k)$. String field operators will be defined for each $[X]$.

A contour $[X]$ is shown in Fig.5. Let the set of links of the form $(\mathbf{x}, 1),(\mathbf{x}, 3)$, but not $(\mathbf{x}, 2)$ below $[X]$ on the triangular lattice be called $\mathcal{D}$. The links of $\mathcal{D}$ are shown as dotted lines in Fig.5. The string-destruction operator at $[X]$ is

$$
\Psi[X]=\left[\prod_{l \varepsilon[X]} \sigma^{-}(l)\right]\left[\prod_{l \varepsilon \mathcal{D}} \sigma^{z}(l)\right]=\left[\sigma^{-}\left(l_{1}\right) \sigma^{-}\left(l_{1}\right) \cdots\right]\left[\prod_{l \varepsilon \mathcal{D}} \sigma^{z}(l)\right] .
$$

Similiarly, the string-creation operator at $[X]$ is

$$
\Psi^{\dagger}[X]=\left[\prod_{l \varepsilon[X]} \sigma^{+}(l)\right]\left[\prod_{l \varepsilon \mathcal{D}} \sigma^{z}(l)\right]=\left[\sigma^{+}\left(l_{1}\right) \sigma^{+}\left(l_{1}\right) \cdots\right]\left[\prod_{l \varepsilon \mathcal{D}} \sigma^{z}(l)\right] .
$$

The string fields satisfy certain anticommutation relations. The $\Psi^{\dagger}$ 's anticommute among themselves, as do the $\Psi$ 's :

$$
\left[\Psi^{\dagger}[X], \Psi^{\dagger}[Y]\right]_{+}=[\Psi[X], \Psi[Y]]_{+}=0,
$$

for any two contours $[X]$ and $[Y]$. A special case of (31) is

$$
\left(\Psi^{\dagger}[X]\right)^{2}=(\Psi[X])^{2}=0 .
$$

In addition to (31), the string fields satisfy

$$
\left[\Psi^{\dagger}[X], \Psi[Y]\right]_{+}=\delta_{[X][Y]}
$$

Suppose $[X]$ and $[Y]$ are two contours which cross at the points $\mathbf{x}, \mathbf{z}$, such that $[X]$ is everywhere below $[Y]$ (Fig.6). By cutting both contours at $\mathbf{x}, \mathbf{z}$, and then 
switching and re-attaching the pieces two new contours, $[Z]$ and $[U]$, are obtained. The string-field $\Psi^{\dagger}$ operators satisfy

$$
\Psi^{\dagger}[X] \Psi^{\dagger}[Y]=(-1)^{z^{2}-x^{2}} \Psi^{\dagger}[Z] \Psi^{\dagger}[U]
$$

Notice that if $[X]$ and $[Y]$ are string contours satisfying the rules of Section 3, the contours $[Z]$ and $[U]$ will not satisfy these rules.

States produced by applying products of operators $\Psi^{\dagger}[X]$ to the zero-string state $\mid Z . S .>$ (satifying $\Psi[X] \mid Z . S .>=0)$ are the second-quantized versions of (26).

For each $[X]$ define a vector $\mid[X]>$, such that $\langle[X]|[X]>=1$ and if $[X] \neq$ $[Y],<[X] \mid[Y]>=0$. These vectors constitute an orthonormal basis of a vector space $V$. The states which correspond to the special string configurations satisfying the rules of Section 3 span a subspace $W$. The mapping $A$ defined in (12) is a linear transformation from $W$ to the spin-chain Hilbert space, which preserves inner products. The Hamiltonian (10), with the constraint (8) enforced, is

$$
\begin{aligned}
H & =-\sum_{\mid[X]>\varepsilon W} \sum_{\mid[Y]>\varepsilon W} \Psi^{\dagger}[Y]<[Y]|\mathcal{H}|[X]>\Psi[Y] \\
& =-\sum_{\mid[X]>\varepsilon W} \sum_{\mid[Y]>\varepsilon W} \Psi^{\dagger}[Y]<[Y]\left|A h A^{\dagger}\right|[X]>\Psi[Y]
\end{aligned}
$$

where $h$ is the spin-chain Hamiltonian defined in equation (14).

The ground state is the antisymmetrized product of ground-state string wave functionals, with the coordinates ordered as discussed previously. By (28) and (24), the ground state $E_{0}$ energy must satisfy

$$
-\frac{1}{\sqrt{3} \pi} J \leq \frac{E_{0}}{N L} \leq-\frac{1}{\pi} J
$$

Correlations in the ground state of an individual string have only power law behaviour, as the spectrum of the spin chain is relativistic and gapless. Thus the ground state of the RKM has power-law correlations, and is neither a columnar nor a staggered state 4 . An excitation of the RKM can be made by exciting a single string. Clearly the gap is zero.

\section{$7 \quad$ Static Defects}

An important issue is how the system behaves when topological defects are introduced. In models of copper-oxide-layer superconductors, defects are charged excitations and their confinement implies Cooper pairing with a short coherence length. Only static defects will be discussed here. The square lattice is bipartite with two geometrically distinct kinds of sites, namely red sites with $x^{1}+x^{2}$ even and black sites with $x^{1}+x^{2}$ odd. Since the Hamiltonian (11) commutes with the Gauss's law operator (2), defect locations are fixed. A red defect becomes a site where a string ends at the 
right, whereas a black defect becomes a site where a string begins at the left. Free boundary conditions for dimers on the square lattice dictate imply that there are an equal number of red and black defects.

An isolated defect on a red site becomes one of the configurations

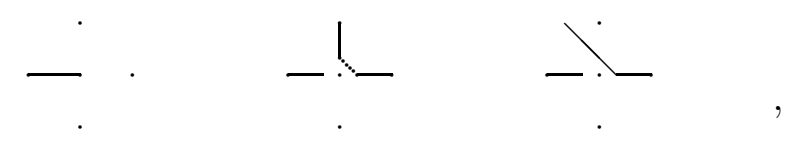

on the triangular lattice, while an isolated defect at a black site becomes one of the configurations

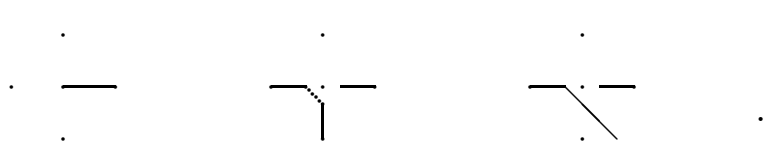

The red defects and black defects behave like positive and negative charges, respectively in a confining $U(1)$ gauge theory 19 . If a string is removed by placing a red defect at the left boundary and a black defect is placed at the right boundary, then the lowest energy state is identical to the ground state with no defects, minus the energy of one string. Since the individual strings have a negative energy per unit length (because the filling fraction in the spin chain is greater than zero), the defects are joined by a physical "string hole" with positive energy per unit length. This energy is minus the ground-state energy of one string. The red defect and the black defect are thus bound by a constant attractive force through the formation of this electric string. The string tension is defined to be the asymptotic energy per unit seperation on the original square lattice. Since the seperation of the defects on the square lattice is $L$, the string tension is $\mu$ (which satisfies the bounds (24). The system should be rotational-invariant at large distances (since the gap is zero) so the string tension will only depend on the seperation of two defects, if that seperation is large.

\section{Conclusions}

It has been shown that the RKM is equivalent to a gas of transversely oscillating "hard" strings. A qualitative analysis shows that the energy spectrum is gapless, indicating a fluid phase. Defects are confined by dynamical strings. Further numerical work on the spin chain (14) describing a single string should reveal further properties, such as the precise values of the string tension and ground-state energy.

The physical excitations are probably closed strings. A closed string excitation is produced by disturbing one of the strings in the ground state (the string "sea") 
along a finite region of its length, then antisymmetrizing and ordering the string coordinates.

It seems likely that for the range of diagonal coupling $J \geq V \geq 0$ there is a fluid phase, with a phase transition to a valence-bond solid for some negative $V$.

\section{Acknowledgements}

I would like to thank Nick Read and Subir Sachdev for educating me on a number of issues and for many useful comments on the manuscript. I am grateful to the the Niels Bohr Institute staff for their hospitality and the Danish Research Council for some financial support.

\section{References}

1. P. Orland, Int. J. Mod. Phys. B5, 2401 (1991).

2. P. Orland, Nucl. Phys. B372, 635 (1992).

3. P. Orland, Phys. Rev. B47, 11280 (1993).

4. D. Rokhsar, S.A. Kivelson, Phys. Lett. 61, 2376 (1988).

5. D.J. Klein, J. Phys. A15, 661 (1982); J.T. Chayes, L. Chayes and S.A. Kivelson, Commun. Math. Phys. 123, 53 (1989).

6. T. Dombre and G. Kotliar, Phys. Rev. B39, 855 (1989); N. Read and S. Sachdev, Nucl. Phys. B316, 609 (1989); Phys. Rev. Lett. 62, 1694 (1989); Int. J. Mod. Phys. B5, 219 (1991).

7. It was incorrecly stated in reference 3 that the authors of reference 6 had made similiar conclusions concerning the RKM.

8. E. Fradkin and S.A. Kivelson, Mod. Phys. Lett. B4, 225 (1990); E. Fradkin, Field Theories of Condensed Matter Systems, Addison-Wesley (1991).

9. S. Sachdev, Phys. Rev. B40, 5204 (1989).

10. N. Read and S. Sachdev, Phys. Rev. B42, 4568 (1990).

11. M.P Gelfand, R.R.P. Singh and D.A. Huse, Phys. Rev. B40, 10801 (1989); M.P. Gelfand, Phys. Rev. B42, 8206 (1990).

12. It was pointed out to me by $\mathrm{N}$. Read that the boundary conditions taken in reference 3 are in fact incompatible with global spin-solid formation. However, it can be shown that the basic results of reference 3 are valid even for boundary conditions not suffering from this difficulty. 
13. Vl.S. Dotsenko and A.M. Polyakov in Advanced Studies in Pure Mathematics 16, ed. M. Jimbo, T. Miwa and A. Tsuchiya, Kinkuniya, Tokyo, 171 (1988); A.R. Kavalov and A. Sedrakyan, Nucl. Phys. B321, 682 (1989); P. Orland, Phys. Rev. Lett. 59, 2393 (1987).

14. D. Horn, Phys. Lett. 100B, 149 (1981).

15. P. Orland and D. Rohrlich, Nucl. Phys. B338, 647 (1990).

16. P.W. Anderson, Science 235, 1196 (1987).

17. Y. Nambu, Progr. Theoret. Phys. 5, 1, E (1950); E. Lieb, T. Schultz and D. Mattis, Ann. Phys. 16, 407 (1961).

18. G. Gómez-Santos, Phys. Rev. Lett. 70, 3780 (1993).

19. A.M. Polyakov, Phys. Lett. 59, 79 (1975); Nucl. Phys. B120, 429 (1977). 


\section{Figure Captions}

- Figure 1 : Reduction of the square lattice to the triangular lattice. The bonds marked with circles are eliminated, converting each square (some of which are labeled by labeled by A, B, C, etc.) to a triangle. For this particular case $N=4$ and $L=7$.

- Figure 2: A typical configuration of the RKM, after reduction to the triangular lattice. Each dimer configuration on the old lattice becomes a set of strings on the new lattice.

- Figure 3 : Two of the four basic four column configurations. By translating (a) horizontally and (b) vertically by one lattice spacing, the other two column configurations are generated.

- Figure 4 : The configurations on the triangular lattice obtained from the column configurations of Fig.3. The other two such configurations are obtained by translating these by one lattice spacing.

- Figure 5: How the Fermionic string fields $\Psi$ and $\Psi^{\dagger}$ are defined for a contour $[X]$. Notice that this particular choice of $[X]$ is not a legitimate string configuration according to the rules of Section 3. The dark lines indicate the links of the string contour $[X]$, while the dotted lines indicate the links in the set $\mathcal{D}$.

- Figure 6 : The relationship between the contours $[X],[Y],[Z]$ and $[U]$. 

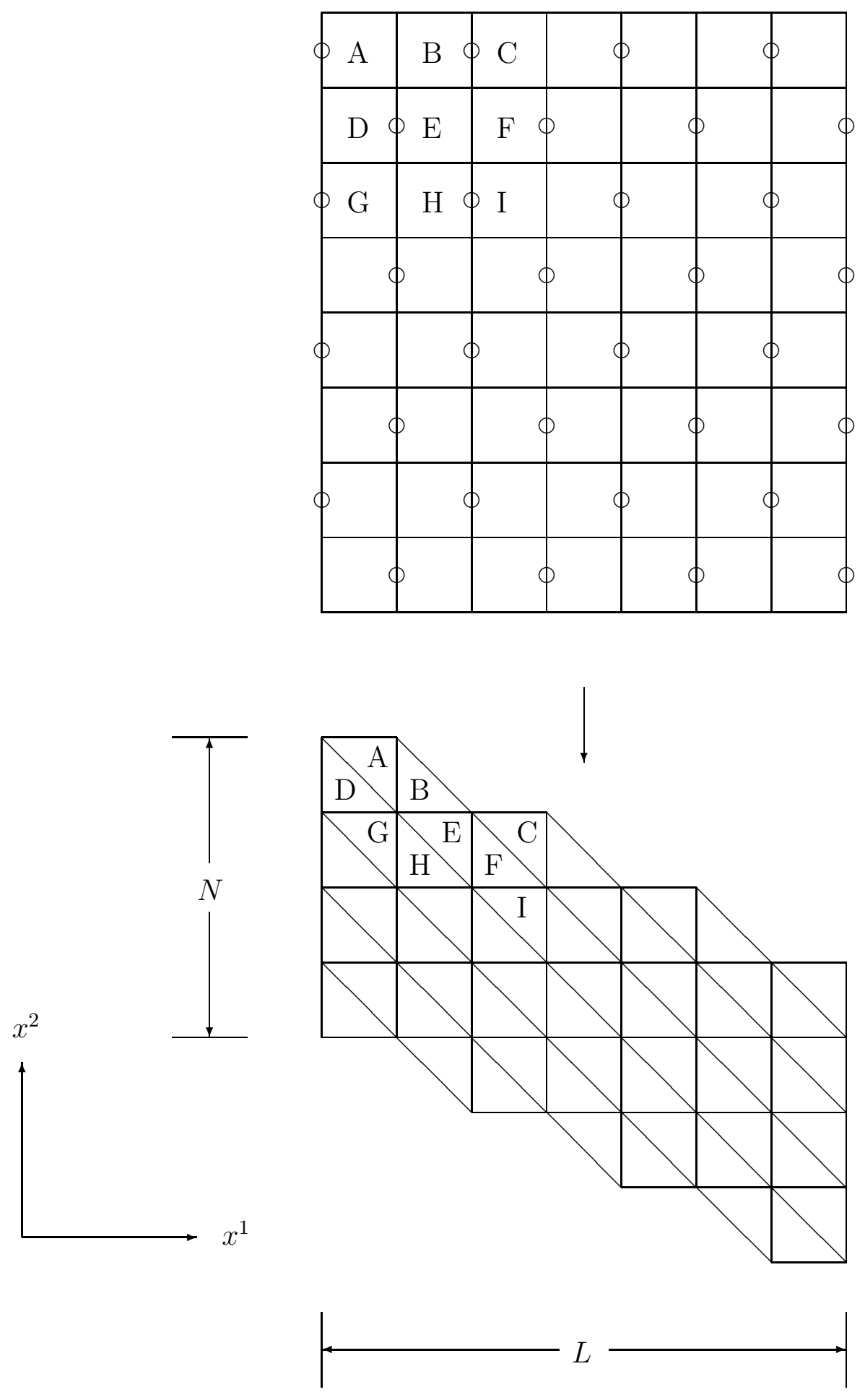

Fig.1 


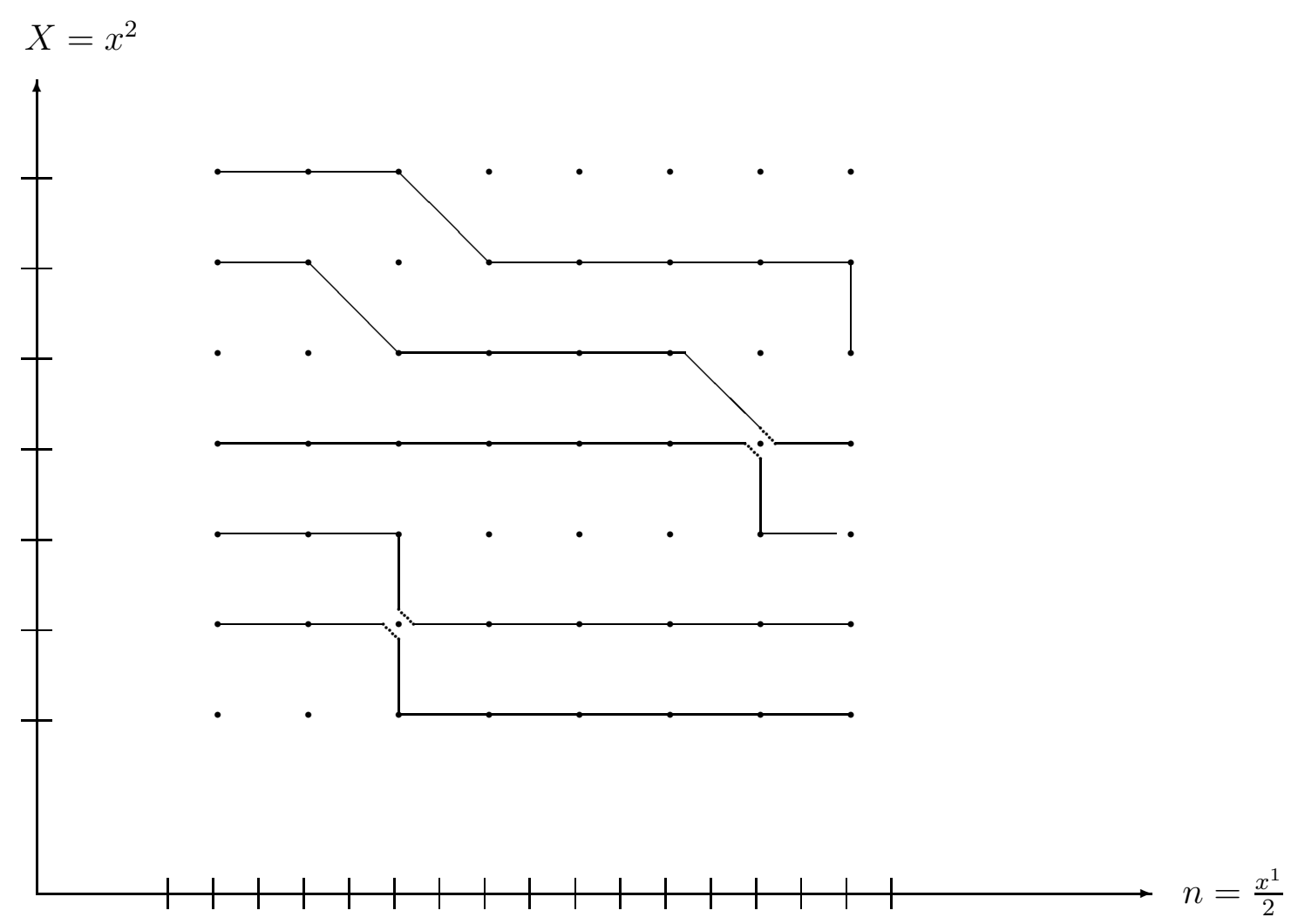

Fig.2 
$=z=z=$
$z=z=z$
$z=z=z$
$z=z=z$
$z=z=z$
$z=z=z$
$z=z=$

(a)

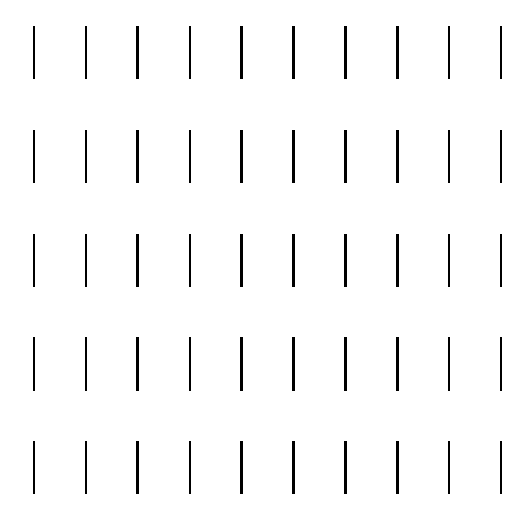

(b)

Fig.3 


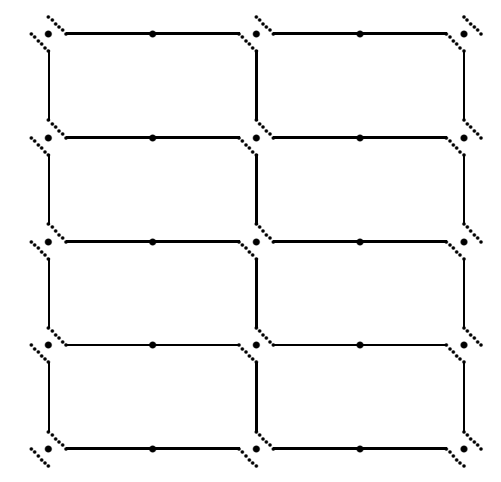

(a)

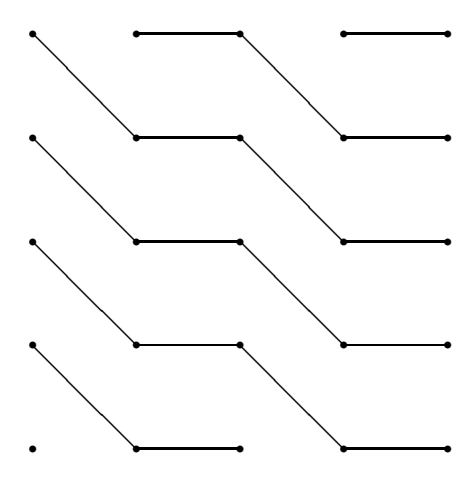

(b)

Fig.4 


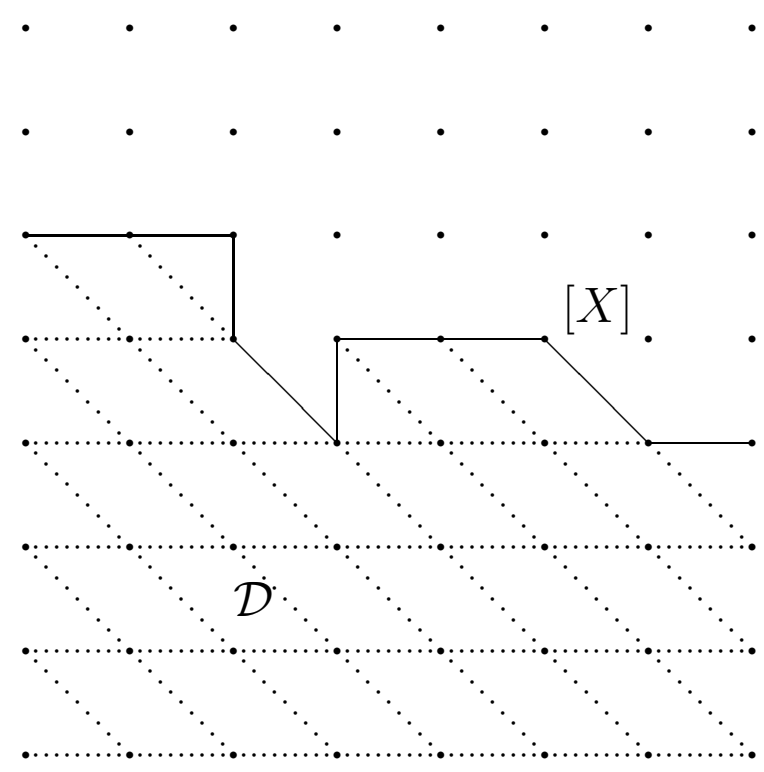

Fig.5 

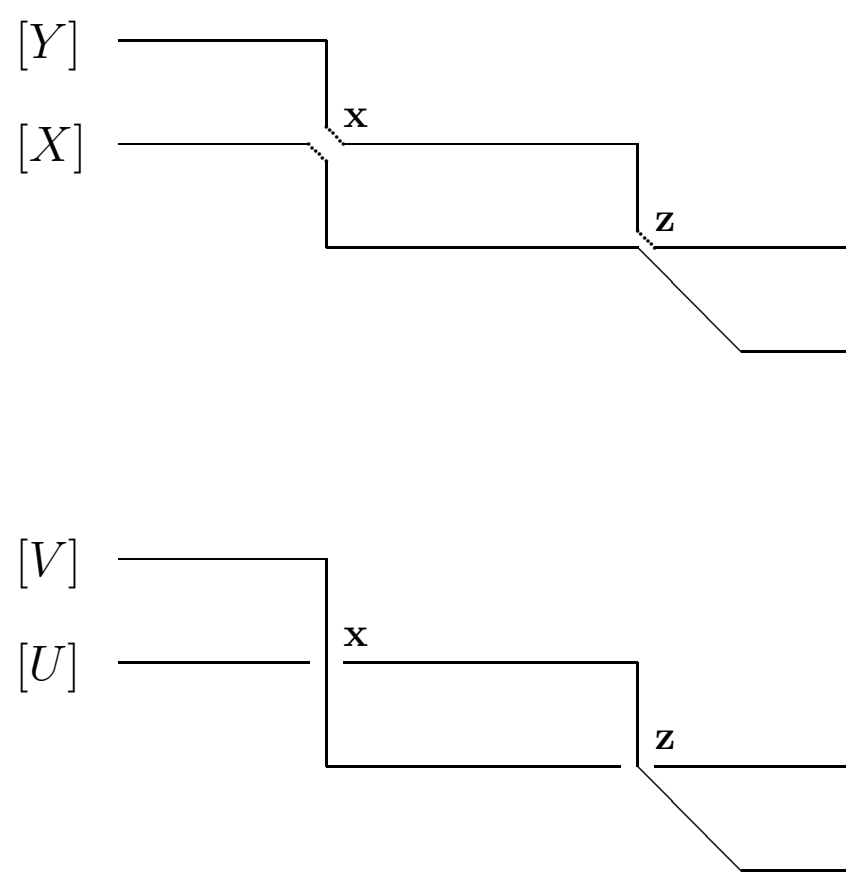

Fig.6 